\section{Necropsy of original case of Lowry's syndrome}

Cataracts, microcephaly, kyphosis, and limited joint movement

SUMMARY The necropsy findings are reported on one of the original sibs of Lowry's syndrome. The child, who died at 7 years of bronchopneumonia, was severely dwarfed, microcephalic, and microphthalmic. The brain was small and extensively calcified, the cerebellar cortex hypoplastic, and the retinae atrophic. There were multiple skeletal and integumental and minor renal abnormalities. The lymphoid tissue was much reduced, and many arteries and arterioles were thickened and narrowed. This constitutes a unique constellation of lesions.

Lowry et al. (1971) described a brother and sister, with cataracts, microcephaly, kyphosis, and limited joint movements, and suggested that they showed a hitherto undescribed autosomal recessive gene syndrome. Both children have since died. No necropsy was done on the boy. The girl lived until the age of 7 years and the necropsy findings are reported here.

\section{Case report}

The details of the girl's early development are described in the article by Lowry et al. (1971). In brief, the child was microcephalic and mentally defective from birth. Needling and aspiration of bilateral cataracts were carried out. A battery of laboratory tests, including karyotype determinations, was normal. The child was in an institution from the age of 3 years. She did poorly for 4 further years, requiring complete care, finally succumbing to pneumonia.

\section{NECROPS Y}

The body presented a grotesque appearance (Fig. 1). It was obese but dwarfed, measuring only $68 \mathrm{~cm}$ crown heel and weighing $7570 \mathrm{~g}$, appropriate for a 6 to 8 months infant. The head circumference was $35 \mathrm{~cm}$

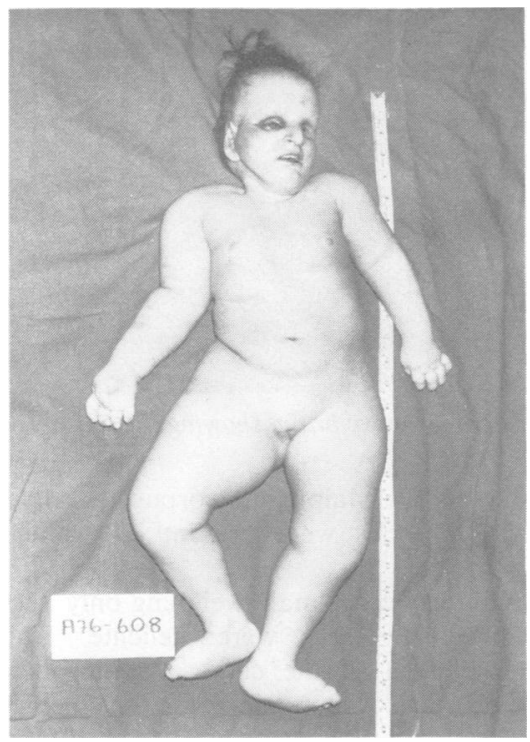

Fig. 1 View of the body at necropsy, aged 7 years.

(at the 50th centile for a newborn). The face had a bird-like appearance. The forehead was prominent, the skull scaphoid with fused sutures, the nose beaked, and the eyes sunken and small. The scalp was thin and the hair very sparse. The mandible was slightly recessed. She had 24 teeth which were poorly enamelled. The upper incisors pointed straight forward. The back was very kyphotic, with a long curve laevoscoliosis. The right hip was dislocated and the leg rotated outwards. The left knee was fixed in $\mathbf{3 0}$ degree flexion. A layer of pale pink-brown fat covered the body. The muscle bulk was generally reduced. The labia majora were slightly hypoplastic.

The cardiovascular and gastrointestinal systems were normal. The respiratory system was anatomically normal but the lungs showed patchy consolidation and were covered by a thin layer of fibrin. The thyroid, adrenal, and pituitary glands were normal. The kidneys were small, weighing 15 and 17.5 $\mathrm{g}$; the expected weight for a child her size would be 62 g combined weight. The shape was normal and there was some fetal lobulation. On section, the number of renal units was decreased, the cortex was thick, and the pyramids small. The thymus was very tiny and weighed only $1.5 \mathrm{~g}$. The spleen was of normal weight 


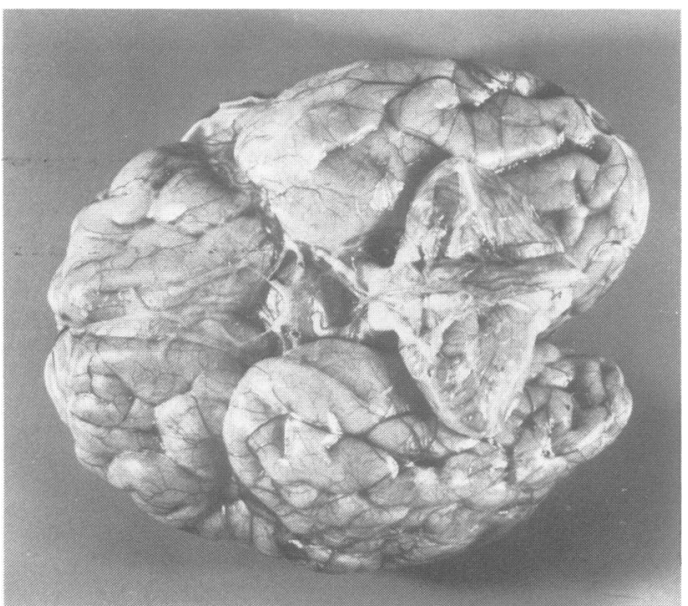

Fig. 2 Inferior view of brain, showing the tiny cerebellum.

for her size but the Malpighian corpuscles could not be seen. Lymph nodes were present but smaller than normal.

The brain was very small, weighing only $360 \mathrm{~g}$. The basal cerebral vessels were delicate. The convolutional pattern of the cerebral hemispheres was normal. A small arachnoid cyst lay between the frontal lobes and had indented the right hemisphere. The cerebellum was particularly small (Fig. 2). A large $\mathbb{\Phi}$ arachnoid cyst, $5 \mathrm{~cm}$ in diameter, covered the dorsal $\mathbb{\Phi}$ aspect of the fourth ventricle. The optic nerves were atrophic and grey, the other cranial nerves appeared $\stackrel{5}{\rightarrow}$ normal. On section, all structures were present, albeit $\overline{0}$ small. The lateral ventricles were dilated and the white $\bar{\sigma}$ matter was somewhat diminished. In the spinal cord $\frac{\bar{\omega}}{5}$ the lateral columns appeared grey. The eyes were $\stackrel{\mathbb{D}}{\Omega}$ microphthalmic. The operation of aspirating the cataracts had resulted in fragmentation of the lenses and adhesions in the anterior chamber. The retinae. were atrophic and somewhat pigmented.

\section{WHOLE BODY RADIOGRAPHS}

The skeletal abnormalities seen externally were confirmed. Premature fusion of the sutures of the skull $\overrightarrow{\mathrm{r}}$ and of the long bone epiphyses was noted. The bones $\omega$ were slender and considerably demineralised.

MICROSCOPICAL EXAMINATION

The lungs showed bronchopneumonia. The lymphoid $\vec{c}$ tissue throughout the body was depleted, with particular involution of the thymus gland, in which also $\mathbb{\Phi}$ the Hassal's corpuscles were degenerated. In the $\vec{\varphi}$ kidney, many glomeruli were ectatic, but the tubules $D$ were normal despite the smallness of the pyramids.

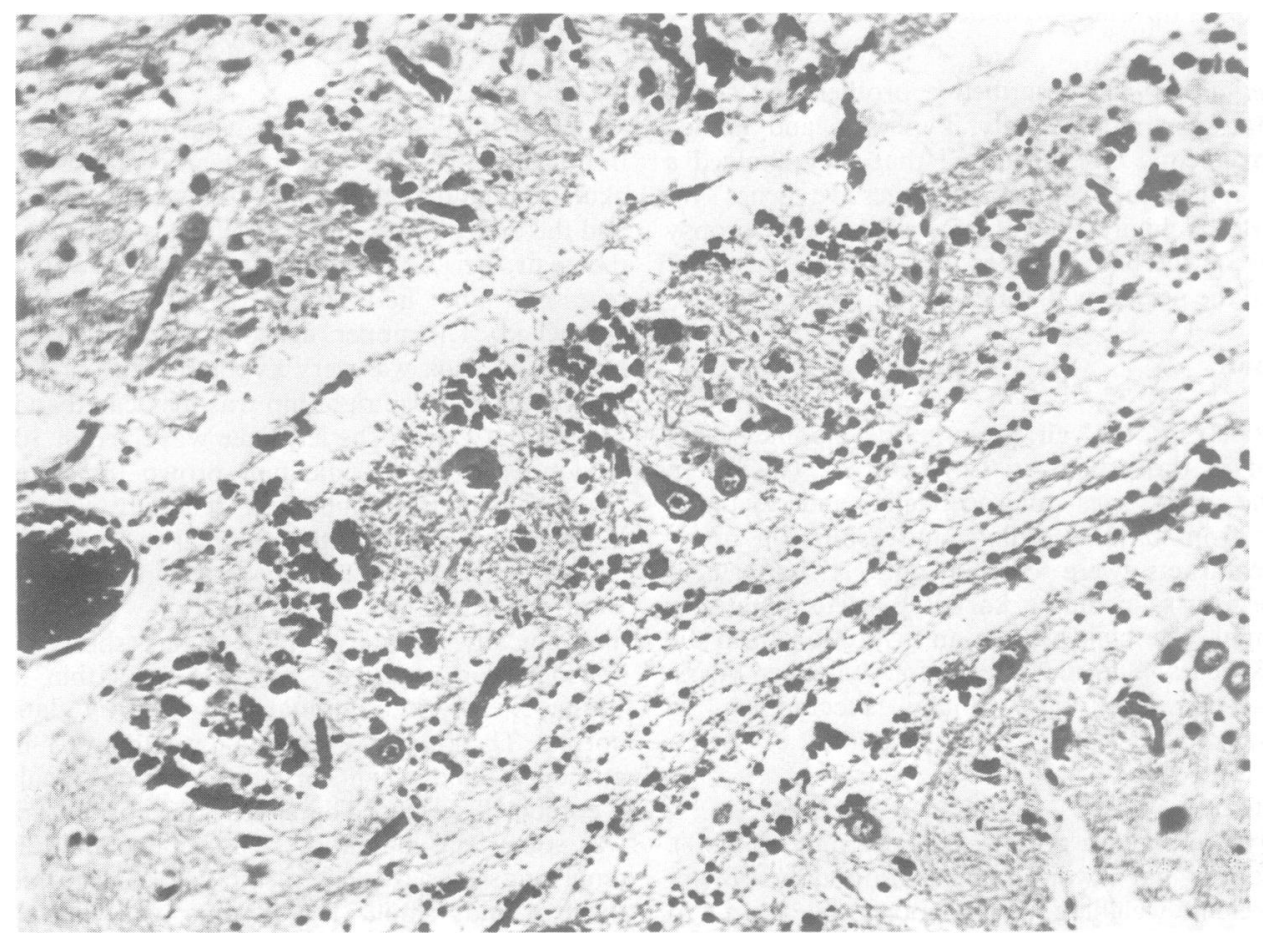

Fig. 3 Hypoplastic, partly calcified, and misaligned cerebellar cortex. (Haematoxylin and eosin. $\times 95$.) 
Many large and smaller arteries in the body, particularly the arcuate vessels in the kidney, the coronary arteries, the aorta, and the carotid vessels, showed intimal thickening. In the aorta, the elastic tissue was somewhat fragmented. In a section through a costochondral junction the growing zone was absent and the bony trabeculae thickened. Samples of muscle showed atrophy and partial replacement by fat. In the skin of the scalp, the hair follicles appeared to be in the resting phase, with little evidence of new hair shaft formation. The epidermis itself seemed inactive. The ' pituitary gland had a large posterior lobe with mucous inclusions.

In the brain, the layering of the cerebral cortex was normal. The white matter was diminished and the corpus callosum was thin and poorly myelinated. The lateral geniculate bodies were severely hypcplastic and the optic radiations virtually absent. The cerebellar cortex was severely hypoplastic (Fig. 3), showing thinning of the molecular layer and a very sparsely populated granule cell layer. The Purkinje cells were best preserved but were frequently displaced out of line and displayed occasional dendritic stars and axonal torpedoes. The inferior olives were normal. The central grey masses in the cerebrum and cerebellum were normally formed. The pyramidal tracts in the brainstem and spinal cord were small and poorly myelinated. A striking feature in the cerebrum and cerebellum was extensive calcification. This was patchy and involved capillaries, the walls of the larger arteries within the brain, and interstitial tissues. The larger arteries often showed narrowing or occlusion of the lumen by fibrous tissue. However, no infarcts had occurred. The calcific deposits were present in cerebrum and cerebellum and were particularly heavy, though in a patchy fashion, in the basal ganglia, cerebellar cortex, and dentate nucleus. In the eyes, the retinae were severely atrophic and disorganised.

\section{Discussion}

Microcephaly with atrophy of the cerebellar cortex may occur in several conditions. It is the significant lesion in viral diseases such as feline panleucopenia and rat virus infections but may also be a nonspecific finding resulting from unknown causes. Some of the features present in this girl have been reported in Cockayne's syndrome, such as dwarfism, kyphosis, microcephaly with atrophy of the cerebellar granule cell layer, retinitis pigmentosa, prognathism of the maxilla, beaked nose, and atrophy of the ovaries. However, other features are quite different. For example, Cockayne's dwarfs never look so bizarre, do not show symptoms until 6 months of age, and survive for decades. Indeed, a comparison of the present case with some of those available in the published reports
(Moossy, 1967) emphasises the differences. We feel, therefore, that one is justified in continuing to recognise the present patient and her brother as having a distinct disease entity, possibly of genetic origin.

We owe the clinical details to Drs B. R. Lowry and B. Tischler. Dr J. Rootman opened the eyes.

\section{L. Dolman and V. J. Wright}

The Department of Pathology, Vancouver General Hospital, and University of British Columbia, Vancouver, BC, Canada

\section{References}

Lowry, R. B., MacLean, R., McLean, D. M., and Tischler, B. (1971). Cataracts, microcephaly, kyphosis, and limited joint movement in two siblings: a new syndrome. Journal of Pediatrics, 79, 282-289.

Moossy, J. (1967). The neuropathology of Cockayne's syndrome. Journal of Neuropathology and Experimental Neurology, 26, 654-660.

Requests for reprints to $\mathrm{Dr}$ C. L. Dolman, Pathology Laboratory, Vancouver General Hospital, 855 West 12th Avenue, Vancouver, B.C., Canada V5Z 1 M9.

\section{Late discovery of a case of testicular feminisation}

SUMMARY The accidental discovery, in an inguinal hernia, of a male gonad in a 67-year-old woman is reported. The association of an unambiguous female phenotype with a purely male karyotype and a male gonad suggests the diagnosis of testicular feminisation. The differential diagnosis, particularly of testicular feminisation with true hermaphroditism, is discussed.

The accidental discovery of a testis during an inguinal hernia repair in a phenotypically female patient raised the differential diagnosis between true hermaphroditism, mixed gonadal dysgenesis, and testicular feminisation. The case is further unusual by its late discovery at the age of 67 years.

\section{Case report}

This 67-year-old woman had been raised as a girl. Her phenotype was female without ambivalence of her external genitalia. At age 22 , she had consulted a 